\title{
Plastic fluctuations in empty crystals formed by cubic wireframe particles
}

DOI:

10.1103/PhysRevMaterials.2.055601

\section{Document Version}

Accepted author manuscript

Link to publication record in Manchester Research Explorer

\section{Citation for published version (APA):}

Mcbride, J., \& Avendano, C. (2018). Plastic fluctuations in empty crystals formed by cubic wireframe particles. Physical Review Materials, 2, [055601]. https://doi.org/10.1103/PhysRevMaterials.2.055601

\section{Published in:}

Physical Review Materials

\section{Citing this paper}

Please note that where the full-text provided on Manchester Research Explorer is the Author Accepted Manuscript or Proof version this may differ from the final Published version. If citing, it is advised that you check and use the publisher's definitive version.

\section{General rights}

Copyright and moral rights for the publications made accessible in the Research Explorer are retained by the authors and/or other copyright owners and it is a condition of accessing publications that users recognise and abide by the legal requirements associated with these rights.

\section{Takedown policy}

If you believe that this document breaches copyright please refer to the University of Manchester's Takedown Procedures [http://man.ac.uk/04Y6Bo] or contact uml.scholarlycommunications@manchester.ac.uk providing relevant details, so we can investigate your claim.

\section{OPEN ACCESS}




\title{
Plastic fluctuations in empty crystals formed by cubic wireframe particles
}

\author{
John M. McBride ${ }^{1}$ and Carlos Avendaño ${ }^{1, *}$ \\ ${ }^{1}$ School of Chemical Engineering and Analytical Science, \\ The University of Manchester, Oxford Road, Manchester M13 9PL, UK.
}

\begin{abstract}
We present a computer simulation study of the phase behaviour of colloidal hard cubic frames, i.e. particles with non-convex cubic wireframe geometry interacting purely by excluded volume. Despite the propensity of cubic wireframe particles to form cubic phases akin to their convex counterparts, these particles exhibit unusual plastic fluctuations in which a random and dynamic fraction of particles rotate around their lattice positions in the crystal lattice while the reminder of the particles remains fully ordered. We argue that this unexpected effect stems from the non-convex geometry of the particles in which the faces of a particle can be penetrated by the vertices of the nearest neighbours even at high number densities.
\end{abstract}

6 One of the triumphs of statistical mechanics in the past 7 century has been the prediction that colloidal particles 8 interacting via short-range repulsive potentials exhibit 9 complex phase behaviour. Beginning with the theoretical 10 studies of Kirkwood [1], subsequent simulations provided 11 the first evidence of the crystallisation of hard spheres [2$124]$. Around the same time, Onsager reported his pioneer${ }_{13}$ ing work to describe the isotropic-nematic phase tran14 sition in systems of thin hard rods and hard discs us15 ing a free-energy functional analysis that depends on the 16 single-particle orientation distribution function [5], yet 17 it took several decades for evidence of the formation of 18 stable smectic and columnar phases using computer sim19 ulation experiments [6-11]. Much early simulation and 20 theoretical work demonstrated that the shape of colloidal ${ }_{21}$ particles is one of the most important features determin22 ing their collective phase behaviour [12-16]. By today we 23 know of a host of crystals, liquid crystals, plastic crystals 24 and quasicrystals formed by hard particles of different 25 shapes and sizes [14, 17, 18].

26 Particular emphasis has been given to the study of ${ }_{27}$ colloidal particles with convex shapes such as spheres, ${ }_{28}$ rods, discs, polyhedra, etc. However, particles with non29 convex geometries have become increasingly important ${ }_{30}$ due to advances in synthetic protocols for the fabrica31 tion particles with complex morphologies and sizes [19$3223]$ and tunable interparticle interactions [24]. Simula33 tions of non-convex particles represent a challenge due 34 to the complex interparticle interactions such as inter35 penetration, shape complementarity, entanglement, and 36 interlocking that can compromise global equilibration. ${ }_{37}$ However, it has been demonstrated recently that these 38 complex interactions can be harnessed to design materi39 als with complex structures [25-33]. For example, simu40 lation studies of rigid planar rings demonstrated that in${ }_{41}$ terpenetration of the particles promotes the unusual for42 mation of stable smectic phases, provided that both inter${ }_{43}$ penetration and symmetry of the rings are high enough ${ }_{44}$ [31]. These liquid crystalline structures are characterised
45 by extremely large free volume since the non-convex rings ${ }_{46}$ are essentially empty, i.e. colloidal rings form smectic ${ }_{47}$ phases with unusually high free volumes. Similarly, ex${ }_{48}$ perimental and simulation studies of branched particles 49 reveal that interlocking effects can promote the formation 50 of ordered superlattices [26].

The aim of this study is to understand the effect of in52 terpenetration in three-dimensional wireframe particles. ${ }_{53}$ In particular, we study the phase behaviour of a particle ${ }_{54}$ model of hard cubic frames (HCFs) interacting only via ${ }_{55}$ excluded volume. Despite the little attention of simula56 tion studies of this kind of particle, the synthesis of three${ }_{57}$ dimensional wireframe particles is not new. More than 20 58 years ago Chen and Seeman reported the synthesis of cu59 bic wireframes using DNA [34]. Today, cubic frames and 60 other wireframe polyhedra are readily synthesised across 61 a range of length scales, from materials as diverse as met62 als [35-39], proteins [40], DNA [41, 42], and polymers and ${ }_{63}$ ceramics [43]. In this paper we use Monte Carlo simu64 lations to demonstrate that HCFs, despite their propen65 sity to form simple cubic crystals similar to their convex ${ }_{66}$ counterparts [44-47], exhibit an unusual type of plastic${ }_{67}$ ity. These cubic crystal phases are characterised by a ${ }_{68}$ high degree of translational and orientational order, but 69 a random and dynamic fraction of particles can rotate 70 even at relatively high densities - i.e. a fraction of the 71 particles are dynamically misaligned with respect to the 72 crystal lattice, an effect which is driven by the partial in73 terpenetration that takes place between a particle in the 74 crystal with its nearest neighbours. We define this be75 haviour as plastic fluctuations, where particles can adopt 76 either aligned or misaligned states separated by a free en77 ergy barrier, and we show that this is indeed an equilib78 rium behaviour. The numerical analysis of the structures 79 reveals that this unusual plasticity is a function of both so the thickness of the HCFs and the density of the system.

\section{METHODS}

We use Monte Carlo (MC) simulations to study the 8s packing and phase behaviour of a HCF particle model, 
${ }_{84}$ which is shown in Figure 1. Similar to a recent model ${ }_{85}$ for tetrahedral frames studied by Ciarella et al. [48], the ${ }_{86}$ edges of each HCF are represented by hard spherocylin${ }_{87}$ ders of diameter $\sigma$ and length $L$. This particle model ${ }_{88}$ allows one to study the effect of frame thickness $t^{*}$ des9 fined as $t^{*}=1 / L^{*}$, where $L^{*}=L / \sigma$ is the aspect ratio 90 of the spherocylinders. Note that in this definition, $L$ ${ }_{91}$ represents the total length of the spherocylinder includ${ }_{92}$ ing the hemispherical caps as shown in Fig. 1(b). In ${ }_{93}$ the limit of $t^{*} \rightarrow 0$ the particles become infinitely thin 94 frames with sharp vertices, while in the limit of $t^{*} \rightarrow 1$ 95 the model essentially becomes a hard sphere of diame96 ter $\sigma$. Since the vertices of the HCFs are represented 97 by hard spheres, particles comprised of short edges be98 come closely related to rounded cubes and superballs for 99 which the phase diagrams have been reported elsewhere $100[49,50]$. In this paper we study frame lengths in the 101 range of $5 \leq L^{*} \leq 100$ which corresponds to a thickness 102 range of $0.01 \leq t^{*} \leq 0.2$. The upper limit $t^{*}=0.2$ is ${ }_{103}$ chosen since frames thicker than this value exhibit quali104 tatively different phase behaviour. An efficient algorithm 105 to detect overlaps between spherocylinders has been re106 ported $[12,51]$, however, special geometrical tests need ${ }_{107}$ to be tailored due to the possibility of unphysical con${ }_{108}$ figurations where frames become interlinked as shown in ${ }_{109}$ Fig. 1(c). These special tests ensure that while an edge of ${ }_{110}$ particle $i$ can penetrate the convex hull of a neighbouring 111 particle $j$, the opposite situation does not occur simulta112 neously since mutual interpenetration of convex hulls by ${ }_{113} \mathrm{HCF}$ edges implies unphysical interlocking. Interparticle 114 interpenetration takes place when the vertex of one HCF ${ }_{115}$ passes through the open facet of another HCF. The po${ }_{116}$ sitions of the eight vertices of a particle $i$ with respect to ${ }_{117}$ the position of its centre of mass $\mathbf{r}_{i}$ is given by

$$
\boldsymbol{\nu}_{i l}=\mathbf{r}_{i}+\frac{L}{2}\left[ \pm \hat{\boldsymbol{e}}_{i 1}, \pm \hat{\boldsymbol{e}}_{i 2}, \pm \hat{\boldsymbol{e}}_{i 3}\right]
$$

${ }_{118}$ where $\boldsymbol{\nu}_{i l}$ is the position of vertex $l$ of particle $i$, and $\hat{\boldsymbol{e}}_{i k}$ 119 is one of the three orthonormal vectors describing the ${ }_{120}$ orientation of particle $i$ in the $k$-direction. Vertex $l$ of ${ }_{121}$ particle $i$ is found to be penetrating a particle $j$ if

$$
\left(\boldsymbol{\nu}_{i l}-\mathbf{r}_{j}\right) \cdot \hat{\boldsymbol{e}}_{j k}<\frac{L}{2}
$$

${ }_{122}$ for each $k=[1,2,3]$. Using these tests, we automatically ${ }_{123}$ reject any $\mathrm{MC}$ move than leads to two or more facets of a ${ }_{124}$ particle $i$ being intersected by edges of a second particle $125 \mathrm{j}$.

${ }_{126}$ Using MC simulations in the isothermal-isobaric NPT ${ }_{127}$ ensemble we explore the phase behaviour of HCFs via ${ }_{128}$ expansion and compression runs allowing the shape of 129 the simulation cell to change. Free energy calculations, ${ }_{130}$ direct coexistence simulations and Gibbs-Duhem integra- ${ }_{146}$ which represents the sum of the fourth-Legendre poly131 tion are also used to determine fluid-solid coexistence 147 nomial evaluated for the cosine of the angles made be${ }_{132}$ point $[11,52-58]$. Properties are reported in dimen- 148 tween $\hat{\boldsymbol{n}}_{1}$ and the three orthonormal vectors $\hat{\boldsymbol{e}}_{i k}$ associ${ }_{133}$ sionless units: pressure $P^{*}=\beta P L^{3}$, number density 149 ated to particle $i$. The vector $\hat{\boldsymbol{n}}_{1}$ is obtained using a

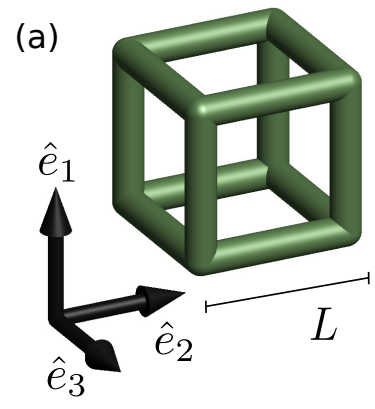

(b)
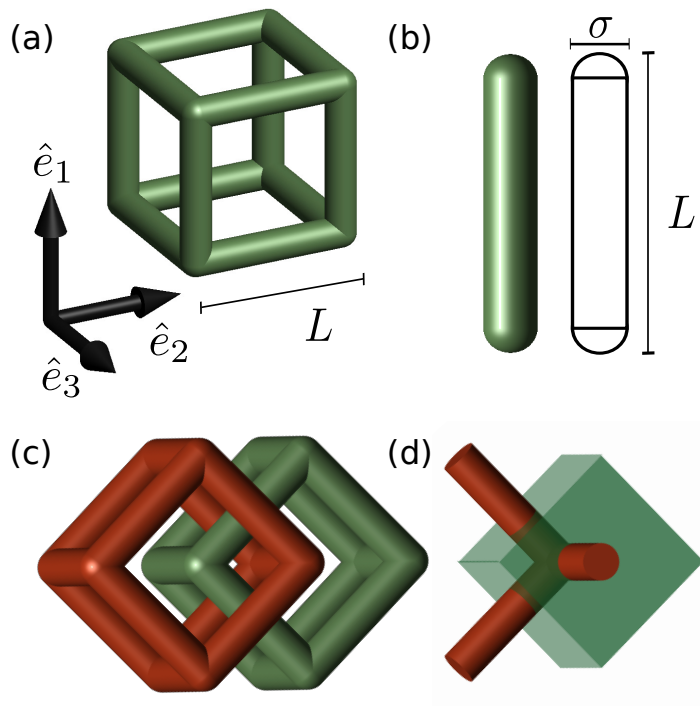

(d)

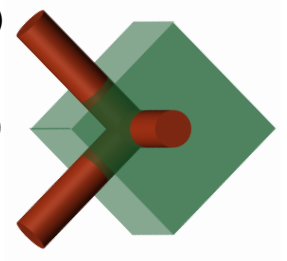

FIG. 1. Schematic representation of the geometry of a hard cubic frame (HCF) of length $L$ and thickness $\sigma / L$. (a) The orientation of a HCF is described by three orthonormal vectors $\hat{\boldsymbol{e}}_{1}, \hat{\boldsymbol{e}}_{2}$ and $\hat{\boldsymbol{e}}_{3}$ that are perpendicular to the empty facets. (b) Each HCF is modelled as sets of 12 spherocylinders of total length $L$ and diameter $\sigma$. A special overlap test has been tailored to avoid unphysical (interlinked) configurations as the one shown in (c). This special test is based on the number of intersection of the edges of the HCFs with the planes of the facets, which in the case of HCFs an unphysical overlap takes place when three facets of a HCF are intersected by three edges of a second particle as shown in (d).

${ }_{134} \rho=L^{3} N / V$, and volume fraction $\phi=v_{f} \rho$, where $P$ 135 is the absolute pressure, $\beta=1 /(k T), T$ is the absolute 136 temperature, $N$ is the total number of HCF particles, $V$ ${ }_{137}$ is the volume of the system, and $v_{f}$ is the volume of a ${ }_{138} \mathrm{HCF}$. The volume $v_{f}$ is given by $v_{f}=3 \pi(L-2 \sigma) \sigma^{2}+8 v_{v}$, 139 where the volume occupied by a vertex $v_{v}=0.8113 \sigma^{3}$ has 140 been calculated using Monte Carlo sampling.

The orientational order of the system is analysed using 142 the cubatic order parameter, $S_{4}$. For this calculation, we ${ }_{143}$ determine the parameter $S_{4,1}$ given by

$$
S_{4,1}=\max _{\hat{\boldsymbol{n}}_{1}} \frac{1}{N} \sum_{i=1}^{N} S_{4,1}^{i}
$$

${ }_{144}$ where $\hat{\boldsymbol{n}}_{1}$ is the vector that maximises $S_{4,1}$, and the local ${ }_{145}$ orientational order parameter $S_{4,1}^{i}$ is defined as

$$
S_{4,1}^{i}=\frac{1}{3} \sum_{k=1}^{3} \frac{1}{8}\left[35\left(\hat{\boldsymbol{e}}_{i k} \cdot \hat{\boldsymbol{n}}_{1}\right)^{4}-30\left(\hat{\boldsymbol{e}}_{i k} \cdot \hat{\boldsymbol{n}}_{1}\right)^{2}+3\right],
$$


150 similar methodology reported in reference 59. A second 202 comprising up to $N=1728$ HCFs. For compression and ${ }_{151}$ indicator of global orientational order is available in $S_{4,2},{ }_{203}$ expansion runs about $10^{6} \mathrm{MC}$ cycles are used for equi${ }_{152}$ which is given by finding $\hat{\boldsymbol{n}}_{2}$, the vector orthogonal to $\hat{\boldsymbol{n}}_{1}{ }_{204}$ libration and another $10^{7} \mathrm{MC}$ cycles are used to collect 153 that maximises $S_{4,2}$. For simple cubic crystals the ensem- ${ }_{205}$ statistics. Each cycle involves $N$ moves with the following ${ }_{154}$ ble averages of $S_{4,1}$ and $S_{4,2}$ are found to be equivalent, 206 probabilities: translation, $47.5 \%$; rotation, $42.5 \%$; assign 155 and for brevity $S_{4,1}$ is reported as $S_{4}$. The distinction 207 a new random orientation, $5 \%$; and volume change, $5 \%$. 156 between the two order parameters becomes useful when ${ }_{208}$ At least 200 configurations are saved for post-processing ${ }_{157}$ considering local orientational order. Using the vector 209 analysis. MC-NVT simulations for systems comprising ${ }_{158} \hat{\boldsymbol{n}}_{1}$ we can find $S_{4,1}^{i}$ using Eq. 4, while $S_{4,2}^{i}$ is calculated ${ }_{210}$ of up to $N=10^{4}$ are also used in order to study the 159 in the same way by replacing $\hat{\boldsymbol{n}}_{\mathbf{1}}$ with $\hat{\boldsymbol{n}}_{\mathbf{2}} . S_{4,1}^{i}$ and $S_{4,2}^{i}{ }^{211}$ plasticity of crystals formed by HCFs in more detail for 160 are found to differ for disordered particles, so the local 212 selected high-density states.

${ }_{161}$ orientational order of particle $i$ is found as the average of ${ }_{213} \quad$ Finally, we have also performed calculations of the po162 the two quantities, i.e.,

$$
S_{4}^{i}=\left(S_{4,1}^{i}+S_{4,2}^{i}\right) / 2 .
$$
214 tential of mean force (PMF) between a pair of particles in 215 isolation using MC simulations. During the simulations, 216 random particle orientations are assigned to the particles ef ${ }_{163}$ As HCFs are essentially empty particles with open ${ }_{219}$ accumulated in small bins. The PMF for a set of values ${ }_{164}$ facets, the degree of interpenetration between a pair of ${ }_{220} r_{i j}$ and $S_{4}^{i j}$ is then obtained as

165 particles is a useful metric that needs to be quantified. A 166 particle interpenetration takes place when a vertex of one ${ }_{167} \mathrm{HCF}$ crosses the open facet of a second particle. Since a ${ }_{168} \mathrm{HCF}$ has 8 vertices, it is hypothetically capable of pene169 trating 8 HCFs at the same time, though we observe that 1706 is the maximum number of interpenetrations for a sin- ${ }^{221}$ 171 gle particle due to steric effects. In this way, we measure 172 the penetration frequency as $\bar{\chi}_{n}=N_{p e n} / N$, where $N_{p e n}{ }_{222}$ We first examine the phase behaviour of HCFs in the 173 is the number of penetrating vertices. If an interpene- ${ }_{223}$ two limiting cases, i.e. $L^{*}=5\left(t^{*}=0.2\right)$ and $L^{*}=100$ 174 tration takes place, we also measure the distance from ${ }_{224}\left(t^{*}=0.01\right)$. The results for the phase behaviour of both 175 the penetrating vertex to the centre of mass of the sec- 225 systems are shown in Fig. 2. For the first case with HCFs 176 ond particle and this is then normalised by dividing by ${ }_{226}$ of $L^{*}=5$, the system exhibits a phase transition from the ${ }_{177}$ the circumscribed radius of a HCF to get $\chi_{d}$. The av- ${ }_{227}$ isotropic phase $\left(\rho=0.55, S_{4} \sim 0\right)$ to a simple cubic (SC) ${ }_{178}$ erage penetration depth $\bar{\chi}_{d}$ is the sum of all $\chi_{d}$ divided ${ }_{228}$ crystal $\left(\rho=0.61, S_{4} \sim 0.72\right)$ at a pressure of $P^{*} \sim 9.52$. 179 by $N_{\text {pen }}$, i.,e. $\bar{\chi}_{d}=N_{p e n}^{-1} \sum \chi_{d}$. Finally to measure the ${ }_{229}$ The large value of $S_{4}$ in the crystal phase denotes at 180 local orientational fluctuations of the system we calculate 230 high degree of orientational order. This value of $S_{4}$ is 181 the fraction $f_{d}$ of misaligned particles with respect to a ${ }_{231}$ similar to the one reported for the $\mathrm{SC}$ crystal formed by ${ }_{182}$ perfect crystal lattice. We consider particle $i$ to be mis- ${ }_{232}$ convex cubes at coexistence where it was observed that ${ }_{183}$ aligned if its local orientational order is below a cut-off, ${ }_{233} S_{4} \sim 0.73$ [44]. It is evident from Figures $2(\mathrm{e}, \mathrm{g})$ that ${ }_{184} S_{4}^{i}<0.6$, and aligned otherwise. This cut-off is chosen ${ }_{234}$ as the density increases the system converges towards 185 based on the minimum observed in histograms of $S_{4}^{i}$ at ${ }_{235}$ perfect order with $S_{4} \rightarrow 1$ and $f_{d} \rightarrow 0$. At high densities 186 various densities where this cut-off clearly separates the ${ }_{236}$ the system undergoes a solid-solid phase transition from ${ }_{187}$ two peaks representing the aligned and misaligned states. ${ }_{237}$ the SC crystal to a sheared simple cubic (ShSC) crystal in ${ }_{188}$ Equations of state are obtained from compression and 238 which the cubic lattice is deformed into a rhombic lattice. 189 expansion runs using MC-NPT simulations. Compres- ${ }_{239}$ The rhombic lattice of this ShSC phase is a consequence 190 sion runs start from a very low density (ideal gas) state 240 of the particle vertices being round, which is an effect 191 and continue up to close-packing. Long simulations are ${ }_{241}$ that is more prominent in thicker HCFs. The formation ${ }_{192}$ required near the coexistence points to allow crystals 242 of ShSC crystals have also been reported for the case of 193 to fully equilibrate. Expansion runs start from either ${ }_{243}$ rounded cubic particles and superballs [50, 61, 62]. 194 a close-packed simple cubic crystal or an equilibrated ${ }_{244}$ The system of HCFs of $L^{*}=100$ also form a similar ${ }_{195}$ crystal configuration. For the simulations involving crys- ${ }_{245} \mathrm{SC}$ crystal as in the previous case, in which the system 196 talline phases, the shape of the box is allowed to change ${ }_{246}$ crystallizes from a fluid state $(\rho=0.55)$ to a SC crystal 197 to aid the relaxation of the lattice by varying the box vec- ${ }_{247}(\rho=0.61)$ at $P^{*}=8.94$. Close to their corresponding 198 tors independently $[52,60]$. A total of $N=216 \mathrm{HCFs}$, ${ }_{248}$ coexistence points, the SC crystals of both $L^{*}=5$ and 199 corresponding to a total of 3072 spherocylinders, are used ${ }_{249} L^{*}=100$ systems exhibit similar structural properties 200 for the calculations of the equations of state. Potential 250 (see Fig. 2 (e-f)), but as the density in the solid phase 201 finite size effects are analysed studying larger systems 251 increases the behaviour diverges considerably. The differ- 
(a)
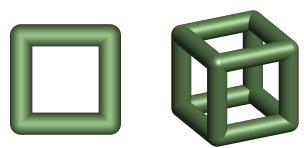

(b)
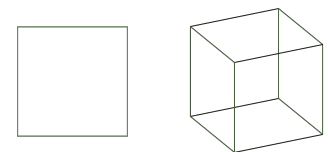

(c)
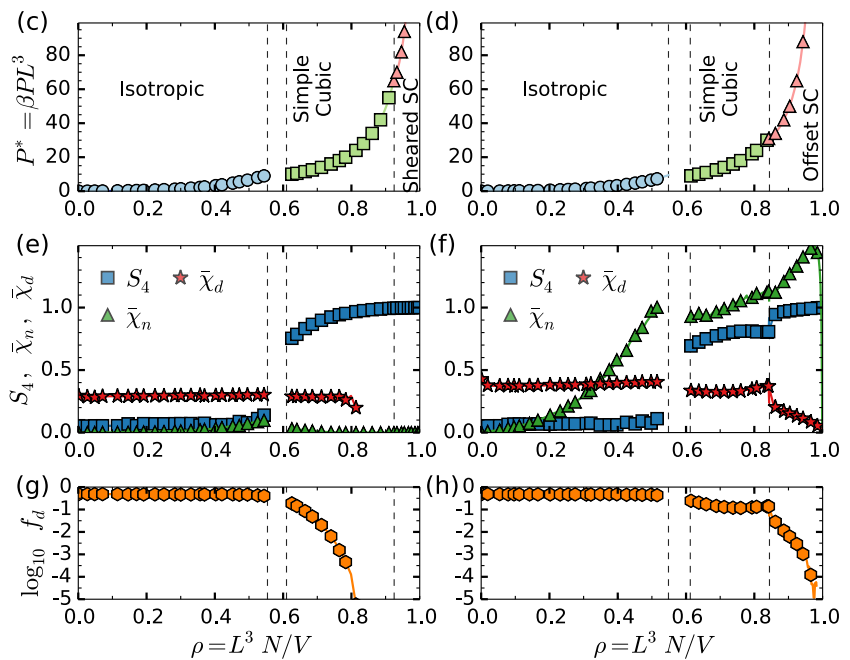

FIG. 2. Representation of hard cubic frames of lengths (a) $L^{*}=5$ and (b) $L^{*}=100$. In (c) and (d), the corresponding equations of state in the $P^{*}-\rho$ representation are presented. In der), $\bar{\chi}_{n}$ (penetration frequency) and $\bar{\chi}_{d}$ (average penetration depth) are shown against number density, $\rho$. The fraction of disordered particles, $f_{d}$, against $\rho$ is also presented in $(\mathrm{g})$ and (h). Vertical dashed lines indicate phase boundaries. (e) and (f) the order parameters $S_{4}$ (global orientational or-

276 phase transition between the SC crystal and an offset 277 simple cubic (OfSC) crystal, which is represented in Fig. 278 3(c). The main difference between both lattices is that in 279 the former the orientations of the particles are on average 280 aligned with the cubic lattice vectors, while in the latter 281 the orientations of the particles are slightly tilted with 282 respect to the cubic lattice. In Figure $2(\mathrm{~h})$ is observed 283 that the fraction of misaligned particles in the OfSC is 284 very small but non-zero indicating that there are still 285 plastic fluctuations occurring up to reduced densities as 286 high as $\rho=0.95$. As the system approaches the limit of ${ }_{287}$ maximum packing both $\overline{\chi_{n}}$ and $\overline{\chi_{d}}$ tend to zero, and at 288 sufficiently high pressures the ShSC phase emerges.

${ }_{289}$ We explore now the structural properties of thin frames ${ }_{290}\left(L^{*}=100\right)$ in more detail to gain more insight into the 291 plastic fluctuations taking place in the crystalline phases. ${ }_{292}$ Three crystalline states are shown in Figure 3 along their 293 orientation distribution plots represented as histograms 294 of the orientations of HCFs projected onto a unit sphere. 295 These orientation distributions describe not only the ori296 entations of high and low probability but also provide 297 information of the rotation mechanism (paths) taken by 298 the particles during the evolution of the simulation. A 299 representative configuration of the SC crystal formed by 300 thin frames near the fluid-solid transition is shown in 301 Fig. 3(a). In this configuration it is observed the parti302 cles prefer to be aligned mainly along the three orthog303 onal axes characterising the cubic phase, but about $18 \%$ 304 of HCFs on average are misaligned. Tracking down the 305 orientations of the misaligned particles shows that these 306 particles eventually realign with respect to the cubic lat307 tice confirming these events are dynamic in nature. As 252 ent order parameters for the system of HCF of $L^{*}=100308$ the density is increased close to the SC-OfSC coexistence 253 are shown in Fig. 2 (f,h) where, in particular, it is ob- 309 we can observe in Fig. 3(b) the particles in the system 254 served that the frequency of interpenetrations, $\bar{\chi}_{n}$, in- 310 seems to be strongly separated into either aligned or mis255 creases with density. Furthermore, the average penetra- 311 aligned configurations. The orientation distribution plot 256 tion depth, $\bar{\chi}_{d}$, appears to have a minimum at $\rho=0.7,312$ now displays a striking network of orientations of higher 257 while as the density increases $S_{4}$ reaches a maximum of 313 or lower probability, indicating that disordered particles ${ }_{258} S_{4}=0.8$ at $\rho=0.79$, and the fraction of disordered 314 rotate along specific routes. These networks, however, 259 particles has a minimum of $f_{d}=0.12$ at $\rho=0.76$. This 315 disappear at sufficiently high densities as shown in Fig. 260 indicates that upon compression, the global orientational $3163(\mathrm{c})$ for the case of the OfSC crystal in which the system 261 order of the systems actually decreases slightly along the 317 is limited to exploring just a few orientations correspond${ }_{262} \mathrm{SC}$ branch. These order parameters show that while the 318 ing to small interpenetration depths. Nonetheless it is 263 system is still highly ordered, there is a large fraction of 319 still remarkable to see these orientational fluctuations at 264 particles which are orientationally misaligned from the 320 a packing fraction close to maximum packing.

${ }_{265}$ crystal lattice. We refer to this phenomenon as plas- ${ }_{221}$ While Fig. 3 gives a simple visual description of the 266 tic fluctuations in which all the particles are predomi- 322 orientational behaviour of the system, a more complete 267 nantly aligned with respect to the crystal lattice, but 323 analysis can be drawn from the set of probability distri268 every particle is still able to rotate and this event takes 324 butions presented in Fig. 4. In Fig. 4(a) we present 269 place spontaneously. Representative configurations for ${ }_{225}$ probability distributions of the local orientational order 270 two SC phases exhibiting a degree of plastic fluctuations 326 parameter, $S_{4}^{i}$, effectively reframing the information used 271 are shown in Figures 3(a-b). While the effect is evident in 327 in the orientation distribution plots. For $\rho \leq 0.85$ there is 272 both snapshots, the clear distinction between aligned and 328 a peak in $\mathcal{P}\left(S_{4}^{i}\right)$ at $S_{4}^{i}=-0.5$, indicating that this level of 273 misaligned particles is more evident in the high-density 329 disorder results in particularly favourable configurations. 274 state shown in Fig. 3(b). Further compression of the ${ }_{330}$ As the density increases to $\rho=0.85, \mathcal{P}\left(S_{4}^{i}\right)$ becomes 275 system to $P^{*}=31$ and $\rho \sim 0.85$ results in a solid-solid 331 strongly bimodal, indicating that particles cross a high 
(a)
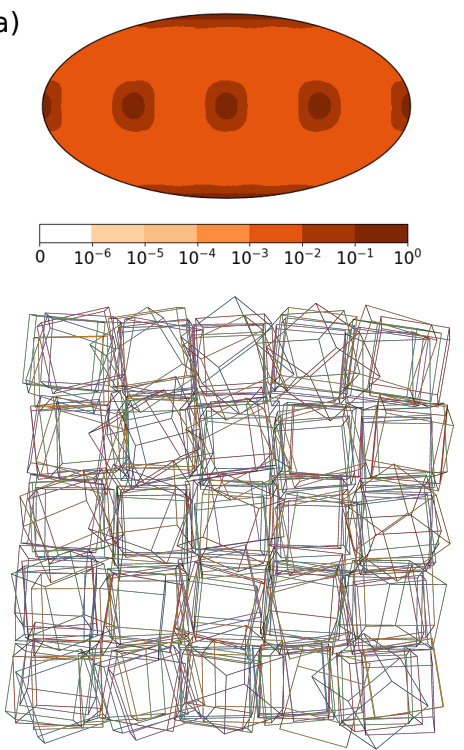

(b)
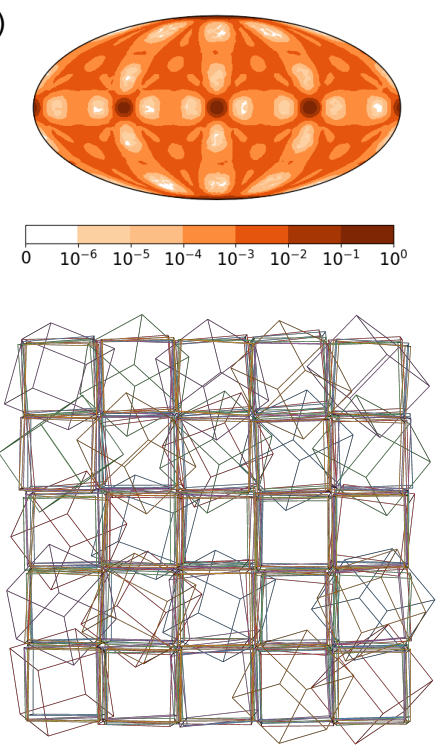

(c)
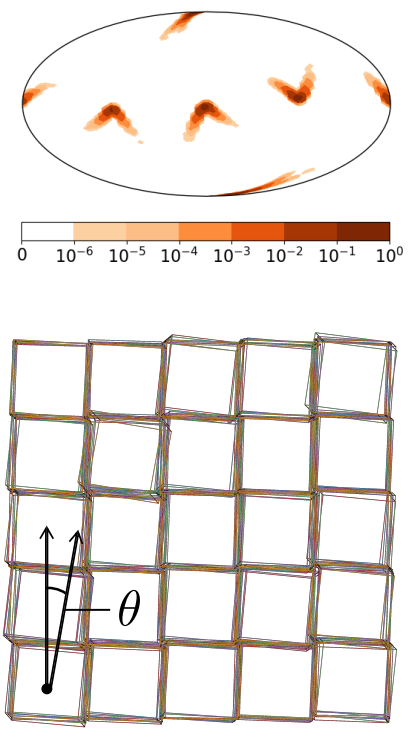

FIG. 3. Snapshots of two representative configuration SC crystals formed by HCF of length $L^{*}=100$ at $\left(\right.$ a) $P^{*}=10(\rho=0.65$, $\left.S_{4}=0.74, f_{d}=0.186\right)$ and (b) $P^{*}=32\left(\rho=0.85, S_{4}=0.79, f_{d}=0.147\right)$, and (c) a representative configuration of an OfSC crystal at $P^{*}=100\left(\rho=0.95, S_{4}=0.99, f_{d}=0.0007\right)$. For each snapshot, orientation distribution plots are displayed in the Mollweide projection; the scale bar indicates the relative probabilities of the orientations adopted by the particles. In (c), $\theta$ is the average angle made between the HCF orientations and the SC unit cell vectors.

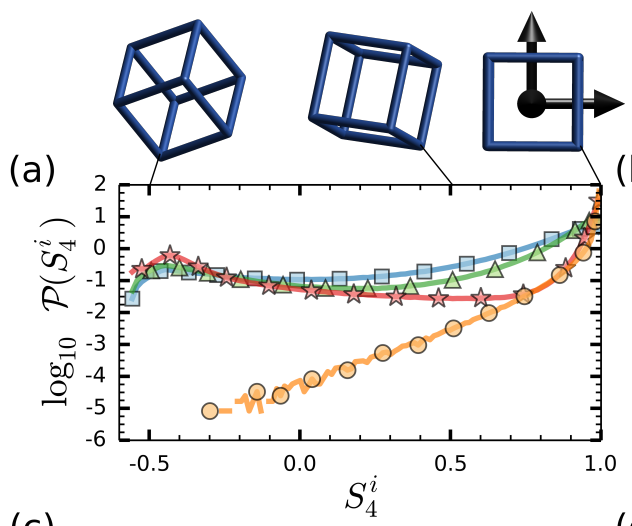

(c)

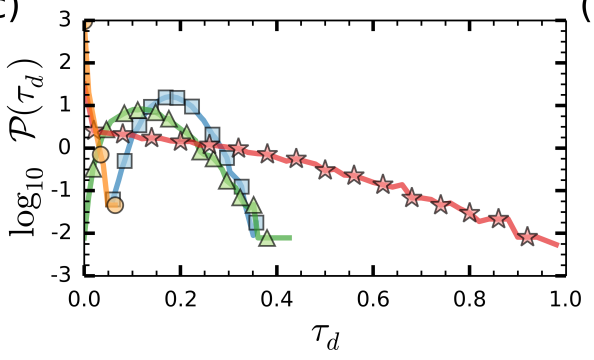

(b)

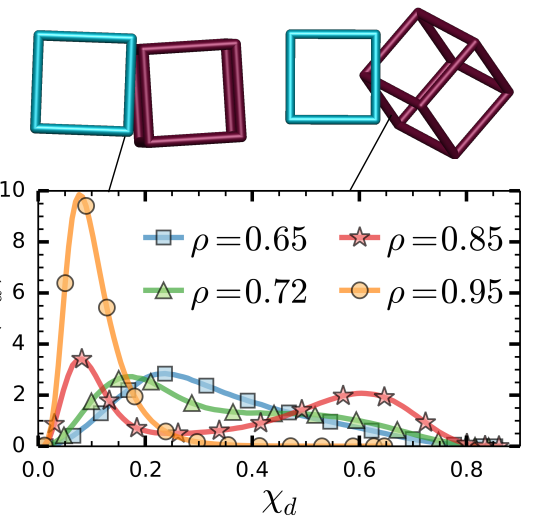

(d)

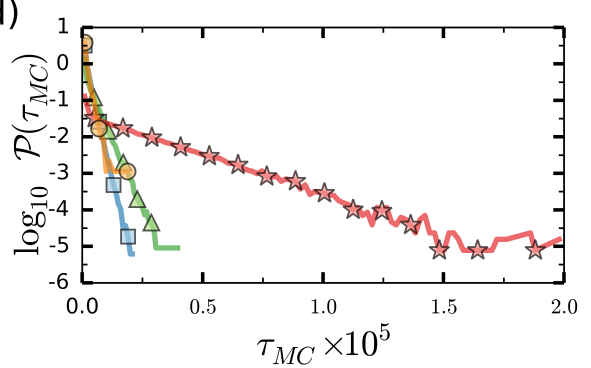

FIG. 4. Probability distributions describing the plastic fluctuations for HCF of $L^{*}=100$ : (a) local orientational order, $S_{4}^{i}$; (b) penetration depth $\chi_{d}$; (c) fraction of time a particle spends in a disordered state within $2 \times 10^{5} \mathrm{MC}$ cycles, $\tau_{d}$; (d) length of time a particle stays in a misaligned state before becoming aligned, $\tau_{M C}$. The results correspond to the states shown in Fig. 3. 
332 free energy barrier to transition from aligned state to mis33 aligned state and vice versa. Potential of mean force cal334 culations for two isolated particles also reveal that HCFs 335 achieve the smallest interparticle distance when one par336 ticle has $S_{4}^{i}=1$ and the other has $S_{4}^{i} \sim-0.5$, which coin337 cides with the first peak observed in $\mathcal{P}\left(S_{4}^{i}\right)$. For $\rho=0.65$ 338 the probability distribution of penetration depths shown 339 in Fig. 4(b) reveals that at lower densities HCFs can pen340 etrate across a wide range of depths. Above $\rho=0.72$, 341 however, $\mathcal{P}\left(\chi_{d}\right)$ also becomes bimodal and by $\rho=0.85$ 342 particles are packed so close that they are only likely to 343 either interpenetrate corner-to-corner (aligned state with 344 low values $\chi_{d}$ ) or interpenetrate deeply (misaligned state 345 with high values of $\chi_{d}$ ). These bimodal probability dis346 tributions explain why plastic fluctuations become even 347 more apparent at higher densities due to the clear dis348 tinction between aligned and misaligned states adopted 349 by the particles. It is important to stress that this be350 haviour is not a result of particles becoming kinetically 351 trapped due to a fast compression; the results presented 352 in Fig. 4 (c-d) were obtained from MC simulations in the ${ }_{353}$ NVT ensemble for systems comprising $N=10^{5} \mathrm{HCFs}$ 354 with perfectly ordered crystals as initial configurations. 355 In order to quantify the dynamic behaviour of particles 356 moving between aligned and misaligned states we present ${ }_{357}$ two measures. The first one is the fraction of time $\tau_{d}$ that 358 a particular HCF spends in the misaligned state, as mea359 sured in a simulation of $2 \times 10^{5} \mathrm{MC}$ cycles - if particles 360 are able to rotate then $\tau_{d}$ should approach the value of $361 f_{d}$ for sufficiently long simulations. The second measure 362 is the length of time $\tau_{M C}$ that a HCF stays in the mis- 3 363 aligned state before switching to an aligned state. For ${ }_{364} \rho=0.65$ Fig. $4(\mathrm{~d})$ shows that HCFs only stay in mis- 3 365 aligned states for short periods of time, and in Fig. 4 (c) 39 ${ }_{366} \mathcal{P}\left(\tau_{d}\right)$ is peaked at the equilibrium fraction of misaligned ${ }_{392}$ ${ }_{367}$ particles, $f_{d}=0.18$. For $\rho=0.85$ the system can still 393 368 switch between states as demonstrated by the broad dis- 394 369 tribution for $\mathcal{P}\left(\tau_{M C}\right)$ in Fig. $4(\mathrm{~d})$, though the dynamics 395 370 are much slower. Given the high free energy barrier be- 396 371 tween the aligned and misaligned states at $\rho=0.85$, if a 372 particle becomes misaligned it remains in that state for 373 a long time.

374 After describing the phase behaviour of the two limit375 ing cases, we proceed to discuss the behaviour of HCF 376 for lengths in the range $5<L^{*}<100$. Fig. 5 (a) 402 377 shows the phase behaviour in the aspect ratio-number 403 378 density plane, $L^{*}-\rho$, which is useful for comparing across 379 the range of $L^{*}$ studied. It is evident that systems of 405 $380 \mathrm{HCFs}$ consistently crystallise from an isotropic fluid to a 406 381 SC crystal at $\rho \sim 0.55$. This means that the fluid-solid 407 382 coexistence observed in HCFs is shifted to higher densi- 4 383 ties compared to convex cubes which start to crystallise 400 384 at $\rho=0.45$. We only observe the appearance of the 410 385 ShSC crystal phase in frames with lengths in the range 4 ${ }_{386} 5 \leq L^{*} \leq 9$, though since it is known that rounded convex ${ }_{387}$ cubes pack most efficiently in the ShSC phase we expect (a)

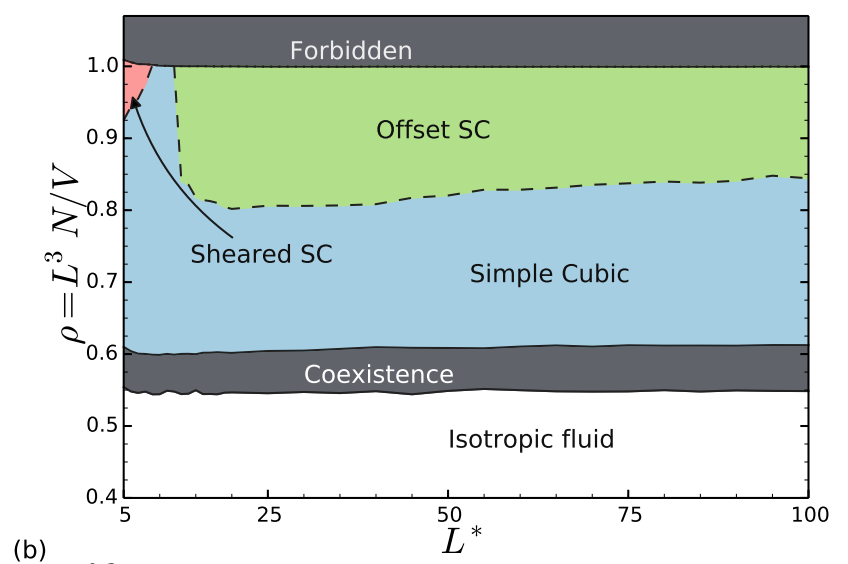

(b)

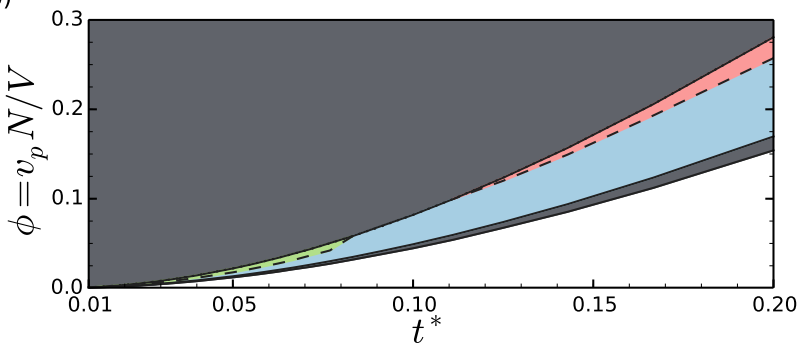

FIG. 5. Phase diagrams of HCFs in (a) the aspect ratio number density $\left(L^{*}-\rho\right)$ plane and in (b) the thickness - volume fraction $\left(t^{*}-\phi\right)$ plane. The colour code indicating the different stable phases in (b) is the same as in (a).

388 that it is stable at maximum packing as long as the $\mathrm{HCF}$ 389 edges are curved [61]. The OfSC phase only becomes 390 stable for $L^{*}>11$ as the HCFs must be thin enough to 91 maintain SC structure while also allowing the corners of 392 HCFs to interpenetrate. Fig. 5 (b) shows the same infor93 mation as Fig 5 (a), but as a function of HCF thickness 394 and volume fraction, $t^{*}-\phi$. As the particles are essentially 395 empty, the volume fraction converges to zero for infinitely 396 thin particles leading to the formation of ordered struc397 tures with unusually high free volumes (porous ordered 398 structures).

399 Finally, it is important to mention that the present 400 study has not considered explicitly the effect of vacan401 cies on the phase behaviour and stability of the ordered 402 phases formed by HCFs. The reports of Smallenburg et. 403 al. [46] and van Damme et. al. [47] showed that vacan404 cies in simple cubic crystals formed by hard convex cubes 405 near the isotropic-solid phase transition enhance the sta406 bility of the crystalline order. Indeed, the results pre407 sented in reference 46 show that vacancies as high as $6 \%$ 408 are observed in the most stable crystals, but this fraction 409 decreases as the density of the system is increased. The 10 results of Smallenburg et. al. [46] suggest that the en411 hanced stability of the vacancy-rich crystal phase is due 42 to the delocalization of the vacancies in the crystals, i.e., 413 vacancies are not strongly bound to a particular lattice 
${ }_{414}$ site thus allowing strings of particles to move coopera- ${ }^{467}$ order. What is striking about this feature of HCFs is the ${ }_{415}$ tively along one of the three orthonormal directions of ${ }_{468}$ range over which plastic fluctuations are evident; that 416 the crystals. We do not expect that vacancies will affect ${ }_{469}$ is, a relatively large fraction of particles continue to be ${ }_{417}$ considerably the results for HCFs presented in this work 470 able to rotate up to number densities of $\rho=0.85$. This ${ }_{418}$ for two reasons. The first reason is due to the effect of ${ }_{471}$ phenomenon is made possible by the wireframe geometry 419 interpenetration itself. The partial interpenetration can 472 - the ability to interpenetrate allows more ways to pack 420 in principle reduce the cooperative diffusion of particles 473 efficiently and rotate which increases the orientational ${ }_{421}$ in a single direction of the crystal since the interpene- 474 entropy of the system. It is important to note that while ${ }_{422}$ trations act as weak "entropic" bonds which will cause 475 hard, rigid particles as thin as $t^{*}=0.01$ are unlikely to ${ }_{423}$ the particles and vacancies to be bound in some degree to 476 be synthesised in the near future, one may observe these ${ }_{424}$ the lattice sites. The second reason is with respect to the 477 plastic fluctuations in HCF particles as thin as $t^{*}=0.1$ 425 difference in the position of the phase coexistence region $478\left(L^{*}=10\right)$.

426 between convex cubes and cubic wireframes. Smallen-

${ }_{427}$ burg et. al. determined that the lowest-density cubic ${ }_{479}$ We would like to thank Dr. David Quigley (Univer${ }_{428}$ crystal phase formed by convex cubes has a density of ${ }_{480}$ sity of Warwick) for his critical comments and sugges${ }_{429} \rho \sim 0.50$ with a vacancy concentration of $6 \%$. The con- ${ }_{481}$ tion of this work. We also want to thank the Computa${ }_{430}$ centration of vacancies is at its highest at this lowest- ${ }_{482}$ tional Shared Facility of the University of Manchester for ${ }_{431}$ density crystal state and decreases to about $0.5 \%$ as the ${ }_{483}$ computing time. JMM acknowledges Doctoral Training ${ }_{432}$ density of the crystal reaches a value of $\rho \sim 0.6$. As re- ${ }_{484}$ Award funding from the University of Manchester.

433 ported in the present study, the lowest density simple cu434 bic phase formed by cubic wireframe particles takes place 435 at a density of $\rho \sim 0.61$ (the phase behavior is shifted to 436 higher densities) thus we expect by simple analogy that ${ }_{437}$ the concentration of vacancies will be extremely low. It 438 is for these two reasons that we conjecture that the role 439 of vacancies in wireframe particles will be less important ${ }_{440}$ than in the case of convex cubes. Nevertheless, additional ${ }_{441}$ simulations considering explicitly vacancies are needed to ${ }_{442}$ probe this argument.

${ }_{444}$ In conclusion, we have studied the phase behaviour ${ }_{497}$ ${ }_{445}$ of particles with cubic wireframe geometry using Monte ${ }_{498}$ ${ }_{446}$ Carlo simulations and a customised overlap algorithm. ${ }_{447}$ We found that as these particles become thinner they 448 increasingly tend to exhibit plastic fluctuations. This 449 behaviour takes place when particles in an ordered crys450 tal spontaneously switch between aligned and misaligned ${ }_{451}$ states. While the dynamics of this switching slows down ${ }_{452}$ as the density is increased, this phenomenon still occurs ${ }_{453}$ at high densities. This behaviour mirrors to an extent the ${ }_{454}$ phase behaviour of convex cubes, where large concentra455 tions of extended vacancies are present near solid-fluid ${ }_{456}$ coexistence, resulting in a fraction of positionally disor${ }_{457}$ dered cubes. One would expect that the absence of solid ${ }_{458}$ facets in the frames would change the phase behaviour 459 of the particles dramatically. However, we observe that 460 in our case the cubic geometry is still the dominant fea461 ture leading to the formation of cubic phases and the ${ }_{462}$ only effect the open facets have on the system is an en463 hancement of the rotational dynamics of the particles ${ }_{464}$ in the solid phases. In other words, the symmetry of 465 the particles control the translational order of the crys466 tal while the open facets control the local orientational ${ }_{522}$

* carlos.avendano@manchester.ac.uk

[1] J. E. Kirkwood, in Phase Transformations in Solids, edited by M. Smoluchowski and Weyl (Wiley, 1951).

[2] B. J. Alder and T. E. Wainwright, J. Chem. Phys. 27, 1208 (1957).

[3] W. W. Wood and J. D. Jacobson, J. Chem. Phys. 27, 1207 (1957).

[4] W. G. Hoover and F. H. Ree, J. Chem. Phys. 49, 3609 (1968).

[5] L. Onsager, Ann. N. Y. Acad. Sci. 51, 627 (1949).

[6] M. Hosino, H. Nakano, and H. Kimura, J. Phys. Soc. Jpn. 47, 740 (1979).

[7] D. Frenkel, H. N. W. Lekkerkerker, and A. Stroobants, Nature 332, 822 (1988).

[8] R. Eppenga and D. Frenkel, Mol. Phys. 52, 1303 (1984).

[9] J. A. C. Veerman and D. Frenkel, Phys. Rev. A 45, 5632 (1992).

[10] S. C. McGrother, D. C. Williamson, and G. Jackson, J. Chem. Phys. 104, 6755 (1996).

[11] P. Bolhuis and D. Frenkel, J. Chem. Phys. 106, 666 (1997).

[12] M. P. Allen, G. T. Evans, D. Frenkel, and B. M. Mulder, Adv. Chem. Phys. 86, 1 (1993).

[13] F. A. Escobedo, Soft Matter 10, 8388 (2014).

[14] G. van Anders, D. Klotsa, N. K. Ahmed, M. Engel, and S. C. Glotzer, Proc. Natl. Acad. Sci. U. S. A. 111, E4812 (2014).

[15] V. N. Manoharan, Science 349, 1253751 (2015).

3 [16] M. A. Boles, M. Engel, and D. V. Talapin, Chem. Rev. 116, 11220 (2016).

[17] S. C. Glotzer and M. J. Solomon, Nat. Mater. 6, 557 (2007).

[18] M. A. Boles, M. Engel, and D. V. Talapin, Chem. Rev. 116, 11220 (2016).

[19] C. J. Hernandez and T. G. Mason, J. Phys. Chem. C 111, 4477 (2007).

[20] S. Sacanna and D. J. Pine, Curr. Opin. Colloid Interface Sci. 16, 96 (2011). 
${ }_{523}[21]$ H. Li, A. G. Kanaras, and L. Manna, Accounts Chem. ${ }_{569}$ ${ }_{524}$ Res. 46, 1387 (2013).

525 [22] S. Sacanna, M. Korpics, K. Rodriguez, L. Colon- 57

528 [23] Y. Xia, K. D. Gilroy, H.-C. Peng, and X. Xia, Angew.

${ }_{530}[24]$ A. Yethiraj and A. van Blaaderen, Nature 421, 513

531

532 [25] C. Avendaño and E. A. Müller, Phys. Rev. E 80, 061702

533

534 [26] M. Marechal, R. J. Kortschot, A. F. Demirors, A. Imhof,

$535 \quad$ and M. Dijkstra, Nano Lett. 10, 1907 (2010).

536 [27] K. Miszta, J. de Graaf, G. Bertoni, D. Dorfs, R. Bres- ${ }_{582}$

538

539

540

541 [29] S. Atkinson, Y. Jiao, and S. Torquato, Phys. Rev. E 86,

542

${ }_{543}$ [30] D. J. Ashton, R. L. Jack, and N. B. Wilding, Phys. Rev.

$544 \quad$ Lett. 114, 237801 (2015).

545 [31] C. Avendaño, G. Jackson, E. A. Müller, and F. A. Es-

${ }_{546}$ cobedo, Proc. Natl. Acad. Sci. U. S. A. 113, 9699 (2016).

547 [32] C. Avendaño and F. A. Escobedo, Curr. Opin. Colloid

$548 \quad$ Interface Sci. 30, 62 (2017).

549 [33] N. Pakalidou, D. L. Cheung, A. J. Masters, and C. Aven-

550 dano, Soft Matter 13, 8618 (2017).

551 [34] J. H. Chen and N. C. Seeman, Nature 350, 631 (1991).

552 [35] X. Lu, L. Au, J. McLellan, Z.-Y. Li, M. Marquez, and

$553 \quad$ Y. Xia, Nano Lett. 7, 1764 (2007).

554 [36] X. Hong, D. Wang, S. Cai, H. Rong, and Y. Li, J. Am. 555 Chem. Soc. 134, 18165 (2012).

556 [37] C. Chen, Y. Kang, Z. Huo, Z. Zhu, W. Huang, H. L. Xin, 6

557 J. D. Snyder, D. Li, J. A. Herron, M. Mavrikakis, et al., $558 \quad$ Science 343, 1339 (2014).

560 I. Gourevich, N. Coombs, and V. Kitaev, J. Am Chem,

561 Soc. 133, 8066 (2011).

562 [39] J. Nai, B. Y. Guan, L. Yu, and X. W. Lou, Sci. Adv. 3, 563 e1700732 (2017).

564 [40] Y.-T. Lai, E. Reading, G. L. Hura, K.-L. Tsai, ${ }_{610}$

565 A. Laganowsky, F. J. Asturias, J. A. Tainer, C. V. Robin-

566 son, and T. O. Yeates, Nat. Chem. 6, 1065 (2014).

567 [41] R. Iinuma, Y. Ke, R. Jungmann, T. Schlichthaerle, J. B.
[42] R. Veneziano, S. Ratanalert, K. Zhang, F. Zhang, H. Yan, W. Chiu, and M. Bathe, Science 352, 1534 (2016).

[43] L. R. Meza, A. J. Zelhofer, N. Clarke, A. J. Mateos, D. M. Kochmann, and J. R. Greer, Proc. Natl. Acad. Sci. U. S. A. 112, 11502 (2015).

[44] U. Agarwal and F. A. Escobedo, Nat. Mater. 10, 230 (2011).

[45] P. F. Damasceno, M. Engel, and S. C. Glotzer, Science 337, 453 (2012)

[46] F. Smallenburg, L. Filion, M. Marechal, and M. Dijkstra, Proc. Natl. Acad. Sci. U.S.A. 109, 17886 (2012).

[47] R. van Damme, B. van der Meer, J. J. van den Broeke, F. Smallenburg, and L. Filion, J. Chem. Phys. 147, 124501 (2017).

[48] S. Ciarella, O. Gang, and F. Sciortino, Eur. Phys. J. E 39, 131 (2016).

[49] R. D. Batten, F. H. Stillinger, and S. Torquato, Phys. Rev. E 81, 061105 (2010).

[50] R. Ni, A. P. Gantapara, J. de Graaf, R. van Roij, and M. Dijkstra, Soft Matter 8, 8826 (2012).

[51] C. Vega and S. Lago, Comput. Chem. 18, 55 (1994).

590 [52] D. Frenkel and B. Smit, Understanding molecular simulation: from algorithms to applications, Vol. 1 (Academic press, 2001).

[53] D. Frenkel and A. J. C. Ladd, J. Chem. Phys. 81, 3188 (1984).

5 [54] B. Widom, J. Chem. Phys. 39, 2808 (1963).

596 [55] A. Fortini, M. Dijkstra, M. Schmidt, and P. P. Wessels, Phys. Rev. E 71, 051403 (2005).

[56] E. G. Noya, C. Vega, and E. de Miguel, J. Chem. Phys. 128, 154507 (2008).

${ }_{00}$ [57] D. A. Kofke, J. Chem. Phys. 98, 4149 (1993).

601 [58] P. J. Camp, C. P. Mason, M. P. Allen, A. A. Khare, and D. A. Kofke, J. Chem. Phys. 105, 2837 (1996).

${ }_{03}$ [59] B. S. John, C. Juhlin, and F. A. Escobedo, J. Chem. Phys. 128, 044909 (2008).

605 [60] R. Najafabadi and S. Yip, Scripta Metall. 17, 1199 (1983).

607 [61] M. Marechal, U. Zimmermann, and H. Löwen, J. Chem. Phys. 136, 144506 (2012).

09 [62] Y. Jiao, F. H. Stillinger, and S. Torquato, Phys. Rev. E 79, 041309 (2009). 